\title{
Characterization, validation, and cross- species transferability of EST-SSR markers developed from Lycoris aurea and their application in genetic evaluation of Lycoris species
}

Yumei Jiang ${ }^{1,2}$, Sheng $X u^{1,2}$, Rong Wang ${ }^{1,2}$, Jiayu Zhou ${ }^{1,2}$, Jian Dou', Qian Yin ${ }^{1}$ and Ren Wang ${ }^{1,2^{*}}$ (D)

\begin{abstract}
Background: The Lycoris genus includes many ornamentally and medicinally important species. Polyploidization and hybridization are considered modes of speciation in this genus, implying great genetic diversity. However, the lack of effective molecular markers has limited the genetic analysis of this genus.

Results: In this study, mining of EST-SSR markers was performed using transcriptome sequences of $L$. aurea, and 839 primer pairs for non-redundant EST-SSRs were successfully designed. A subset of 60 pairs was randomly selected for validation, of which 44 pairs could amplify products of the expected size. Cross-species transferability of the 60 primer pairs among Lycoris species were assessed in L. radiata Hreb, L. sprengeri Comes ex Baker, L. chinensis Traub and $L$. anhuiensis, of which between 38 to $77 \%$ of the primers were able to amplify products in these Lycoris species. Furthermore, 20 and 10 amplification products were selected for sequencing verification in $L$. aurea and $L$. radiata respectively. All products were validated as expected SSRs. In addition, 15 SSRs, including 10 sequenceverified and 5 unverified SSRs were selected and used to evaluate the genetic diversity of seven L. radiata lines. Among these, there were three sterile lines, three fertile lines and one line represented by the offspring of one fertile line. Unweighted pair group method with arithmetic mean analysis (UPGMA) demonstrated that the outgroup, L. aurea was separated from L. radiata lines and that the seven L. radiata lines were clustered into two groups, consistent with their fertility. Interestingly, even a dendrogram with 34 individuals representing the seven $L$. radiata lines was almost consistent with fertility.

Conclusions: This study supplies a pool of potential 839 non-redundant SSR markers for genetic analysis of Lycoris genus, that present high amplification rate, transferability and efficiency, which will facilitate genetic analysis and breeding program in Lycoris.
\end{abstract}

Keywords: EST-SSR, Molecular markers, Genetic analysis, Polymorphism, Lycoris

\footnotetext{
* Correspondence: rwang@cnbg.net

${ }^{1}$ Jiangsu Key Laboratory for the Research and Utilization of Plant Resources,

Institute of Botany, Jiangsu Province and Chinese Academy of Sciences,

Nanjing 210014, China

${ }^{2}$ The Jiangsu Provincial Platform for Conservation and Utilization of

Agricultural Germplasm, Nanjing 210014, China
}

(C) The Author(s). 2020 Open Access This article is licensed under a Creative Commons Attribution 4.0 International License, which permits use, sharing, adaptation, distribution and reproduction in any medium or format, as long as you give appropriate credit to the original author(s) and the source, provide a link to the Creative Commons licence, and indicate if changes were made. The images or other third party material in this article are included in the article's Creative Commons licence, unless indicated otherwise in a credit line to the material. If material is not included in the article's Creative Commons licence and your intended use is not permitted by statutory regulation or exceeds the permitted use, you will need to obtain permission directly from the copyright holder. To view a copy of this licence, visit http://creativecommons.org/licenses/by/4.0/ The Creative Commons Public Domain Dedication waiver (http://creativecommons.org/publicdomain/zero/1.0/) applies to the data made available in this article, unless otherwise stated in a credit line to the data. 


\section{Background}

The genus Lycoris, a member of the Amaryllidaceae family, contains 30 species distributed all over the world. In China, there are 15 species and 1 variety, among which, $L$. radiata and $L$. aurea are the most widespread species. Species of Lycoris genus are used as medical herbs that produce unique amaryllidaceae alkaloids, which exhibit a wide range of medical functions, such as anti-viral, antitumor and acetyl-cholinesterase-inhibitory activities [1-4]. Moreover, since flowers of this genus have a variety of colors and shapes, and some also have fragrance [5], they are very popular as ornamental plants. Hence the demand for Lycoris species has been increasing.

Polyploidization and hybridization are considered modes of speciation in this genus [6-9]. Karyotype investigation has been widely performed in Lycoris, and many karyotypes, such as $2 \mathrm{n}=12,13,14,15,16,17,18$, $19,21,22,24,25,27,30,32,33,44$ have been found, corresponding to a status of diploid, aneuploid, triploid, and tetraploid respectively [7, 8, 10-13]. The basic chromosome number of Lycoris varies from $\mathrm{x}=6$ to $\mathrm{x}=$ 11 [5], while $\mathrm{x}=8$ and $\mathrm{x}=11$ are the main types. For example, L. chinensis, L. anhuiensis and L. longituba are species with $\mathrm{x}=8$, and $L$. radiata, $L$. sprengeri and $L$. sanguinea are species with $\mathrm{x}=11[6,8]$. Moreover, some species originated from hybridization between two species with $\mathrm{x}=11$, or between species with $\mathrm{x}=8$, and with $\mathrm{x}=11$. For example, $L$. straminea is a hybrid between $L$. radiata var. pumila and L. chinensis $[6,14]$. Flow cytometry analysis demonstrated that genome sizes of species with $\mathrm{x}=11$ and $\mathrm{x}=8$ differ greatly and even variation was observed among lines of $L$. radiata [15]. Based on these observations, Lycoris species are expected to have high genetic diversity. Thus, genetic analysis of Lycoris is important not only for uncovering the origin of Lycoris species, but also for efficient conservation and development of elite germplasm.

Molecular markers have proven to be effective in the analysis of genetic diversity. Many types of DNA-based molecular markers have been employed in the analysis of genetic diversity, such as RFLPs, RAPDs and SSRs $[16,17]$, among which SSRs are very popular for their co-dominant inheritance, good reproducibility and costeffectiveness $[18,19]$. SSRs are developed from two types of sequence resources. One is from genomic DNA, called genomic SSRs, and the other is from expressed sequence tags (ESTs), called EST-SSRs or genic SSRs. Compared to genomic SSRs, EST-SSRs are more transferable across related species [20]. Nowadays, more and more species have been sequenced using Next Generation Sequencing techniques, thus a large number of referable ESTs are available in GenBank. Therefore, developing EST-SSR markers will be convenient for many species, especially for those whose genomic researches progress slowly. For example, EST-SSR markers have been developed from transcriptome data of Curcuma alismatifolia [21], Dendrobium officinale [22], Salix psammophila [23] and Robinia pseudoacacia [24].

In Lycoris, transcriptome sequencing has been performed in L. longituba, L. aurea and L. sprengeri [25-28], and abundant sequences containing SSRs have been obtained, which offer a convenient and cost-effective opportunity to develop EST-SSRs. Nonetheless, there have been only 27 EST-SSRs developed from L. longituba [25] and L. sprengeri [29], and 27 genomic SSRs from $L$. radiata, as well as a hybrid between $L$. aurea and $L$. radiata $[30,31]$. Thus, developing more SSRs in Lycoris genus is demanded for genetic analysis and for facilitating research on germplasm conservation and breeding. Previously, using short reads sequencing technology (Illumina), we sequenced the transcriptome of $L$. aurea seedlings subjected to methyl jasmonate (MJ) treatment and assembled 59,643 unigenes [27]. In this study, EST-SSR mining was carried out based on these data. The major objective of this study was to supply a vast pool of non-redundant SSR markers for the genetic analysis of the Lycoris genus and to facilitate the development and utilization of elite germplasm in this genus.

\section{Results \\ Development and characterization of EST-SSRs}

Among the 59,643 unigenes assembled from transcriptome sequences of L. aurea [27], a total of 4637 SSRs were detected using the MISA program. To eliminate the redundant SSRs with respect to alternative transcripts of the same gene, sequence alignment was performed with BioEdit. Finally, primers were designed for a total of 839 SSRs including 623 tri-nucleotide repeats, 147 di-nucleotide repeats, 9 tetra-nucleotide repeats, 13 penta-nucleotide repeats, and 47 hexa-nucleotide repeats. As shown in Table 1, tri- and di-nucleotide repeats were main types of the detected SSRs, which was consistent with a previous report from an analysis using 454 pyrosequencing [26]. Our data showed that tri-nucleotide repeats accounted for $74.25 \%$, in which (AAG)n/(CTT) n was the most common type. In addition, di-nucleotide repeats accounted for $17.52 \%$, in which $(\mathrm{AG}) \mathrm{n} /(\mathrm{CT}) \mathrm{n}$ repeat was the most common type, and $(\mathrm{GC}) \mathrm{n} /(\mathrm{GC}) \mathrm{n}$ was the least common. Among the EST-SSR sequences, there were 28 ESTs containing 2 SSR loci, 4 ESTs containing 3 SSR loci and 1 EST containing 8 SSR loci. In all, a total of 839 pairs of SSR-specific primers were designed using software Primer3 (http://bioinfo.ut.ee/ primer3/), representing 796 unigenes (Table S1).

\section{Validation of EST-SSRs}

To validate the designed primers, a subset of 60 primer pairs was randomly selected and tested in $L$. aurea, which consisted of 9 di-nucleotide repeats, 46 trinucleotide repeats, 1 tetra-nucleotide repeats, 2 penta- 
Table 1 Frequency of di- to hexa-nucleotide repeat motifs in $L$. aurea

\begin{tabular}{|c|c|c|c|}
\hline Types & Repeat Motifs & Numbers & Total \\
\hline \multirow[t]{4}{*}{ Dinucleotide } & $\mathrm{AC/GT}$ & 40 & 147 \\
\hline & $\mathrm{AG} / \mathrm{CT}$ & 77 & \\
\hline & AT/AT & 26 & \\
\hline & $\mathrm{CG} / \mathrm{CG}$ & 4 & \\
\hline \multirow[t]{18}{*}{ Trinucleotide } & AAC/GTT & 38 & 623 \\
\hline & $\mathrm{AAG} / \mathrm{CTT}$ & 116 & \\
\hline & AAT/ATT & 20 & \\
\hline & ACC/GGT & 36 & \\
\hline & ACG/CGT & 32 & \\
\hline & ACT/AGT & 35 & \\
\hline & $\mathrm{AGC/GCT}$ & 41 & \\
\hline & $\mathrm{AGG} / \mathrm{CCT}$ & 47 & \\
\hline & ATC/GAT & 37 & \\
\hline & ATG/CAT & 26 & \\
\hline & $\mathrm{CTG} / \mathrm{CAG}$ & 45 & \\
\hline & CCG/CGG & 39 & \\
\hline & TGT/ACA & 7 & \\
\hline & TCT/AGA & 33 & \\
\hline & TAT/ATA & 5 & \\
\hline & $\mathrm{GAG} / \mathrm{CTC}$ & 41 & \\
\hline & GCG/CGC & 12 & \\
\hline & GTG/CAC & 13 & \\
\hline \multirow[t]{6}{*}{ Tetranucleotide } & AAGA & 1 & 9 \\
\hline & TITA/AAAT & 3 & \\
\hline & ATCA & 1 & \\
\hline & TTAT/AATA & 2 & \\
\hline & ACAT & 1 & \\
\hline & CATA & 1 & \\
\hline \multirow[t]{7}{*}{ Pentanucleotide } & AAGAA & 1 & 13 \\
\hline & ATTाT/AAAAT & 4 & \\
\hline & AATTC & 1 & \\
\hline & AAAAG/TITC & 2 & \\
\hline & AAATA/TATTT & 3 & \\
\hline & AGTGC & 1 & \\
\hline & CCTCG & 1 & \\
\hline Hexanucleotide & ns & ns & 47 \\
\hline
\end{tabular}

Note: $n s$ not showed

nucleotide repeats and 2 hexa-nucleotide repeats. Fortyfour primer pairs produced clear bands that matched the predicted sizes, accounting for $73.3 \%$ effective amplification. To confirm whether the amplification products are SSRs, 20 amplification products observed as clear and strong bands on a high-resolution agarose gel were selected, cloned and sequenced (Table S6). The results showed that the repeat motifs were consistent with the predicted sequences for all the 20 SSRs, though some SSRs varied a little in the repeat numbers. As shown in Table 2, the repeats in LaES12, LaES18, LaES25, LaES36 and LaES41 were reduced, whereas those in LaES20, LaES22, LaES31, and LaES53 were increased, while the others kept consistency with the predicted repeat number. Furthermore, the 20 sequence-verified SSRs were employed for polymorphic analysis of $11 \mathrm{~L}$. aurea individuals (Table S2). One hundred clear bands were amplified and $85 \%$ of them were polymorphic. The results demonstrated that these EST-SSRs are useful for genetic analysis in $L$. aurea.

\section{Transferability of the EST-SSR markers within the Lycoris genus}

To verify whether the primer pairs designed from the EST sequences of $L$. aurea could also effectively amplify the same SSR motifs in other Lycoris species. $L$. radiata and $L$. sprengeri with $\mathrm{x}=11$ as well as $L$. chinensis and $L$. anhuiensis with $\mathrm{x}=8$, were selected for analysis. As shown in Table 3, 44 of the 60 EST-SSRs amplified products of expected size in $L$. aurea and $L$. radiata, while 45, 23 and 30 EST-SSRs were amplifiable in $L$. sprengeri, L. chinensis and $L$. anhuiensis respectively, accounting for $38-77 \%$ amplification rate. These results demonstrated that amplification rates differed greatly in the assessed species, high in $L$. radiata and $L$. sprengeri, and low in L. chinensis and L. anhuiensis. Additionally, two markers, LaES23 and LaES24 with no amplification products in $L$. aurea, produced amplicons in $L$. radiata and/or $L$. sprengeri, indicating that an insertion may have occurred in this region of $L$. aurea.

Further sequencing verification was performed to validate whether such amplification products correspond to the same SSRs in these Lycoris species. As shown in Table 2, ten of the 20 sequence-verified SSRs in L. aurea were selected and confirmed in L. radiata. Moreover, LaES36 and LaES53 were validated in all the five species (Table S6). In general, these repeat motifs were consistent among the Lycoris species, although there was a small difference in the number of repeat motifs. In the ten SSRs generated for $L$. radiata, the repeat motifs decreased in five SSRs, increased in two and were unchanged in the remaining three SSRs (Table 2). Meanwhile the sequences of LaES36 and LaES53 from all five species were aligned. As shown in Fig. 1, multiple sequence deletions were observed in the amplification of LaES36 in L. radiata, but the flanking sequences of the repeat motifs, designed as primer sequences, were conserved in all the five species. Conservation of the SSR flanking sequences would account for the transferability of EST-SSRs [20]. Amplification products subjected to sequence verification were all validated as SSRs, which 
Table 2 Accuracy and authenticity of EST-SSRs confirmed by sequencing

\begin{tabular}{|c|c|c|c|c|c|c|}
\hline \multirow[t]{2}{*}{ Markers } & \multirow{2}{*}{$\begin{array}{l}\text { Repeats } \\
\text { predicted }\end{array}$} & \multicolumn{5}{|c|}{ Repeats in } \\
\hline & & L. aurea & L. radiata & L. sprengeri & L. chinensis & L. anhuiensis \\
\hline LaES3 & $(G C A)_{5}$ & $(G C A)_{5}$ & / & / & / & / \\
\hline LaES6 & $(\mathrm{CTT})_{7}$ & $(\mathrm{CTT})_{7}$ & / & / & / & / \\
\hline LaES12 & $(G A)_{8}$ & $(G A)_{6}$ & / & / & I & / \\
\hline LaES13 & $(C G C)_{6}$ & $(C G C)_{6}$ & $(C G C)_{4}$ & / & / & / \\
\hline LaES18 & $(\mathrm{GAA})_{5}$ & $(\mathrm{GAA})_{4}$ & $(\mathrm{GAA})_{5}$ & / & / & / \\
\hline LaES20 & $(\mathrm{GAA})_{5}$ & $(\mathrm{GAA})_{8}$ & / & / & - & / \\
\hline LaES22 & $(A G G)_{4}$ & $(A G G)_{5}$ & $(A G G)_{3}$ & / & / & / \\
\hline LaES25 & $(\mathrm{TTCTT})_{4}$ & $(\mathrm{TTCTT})_{3}$ & $(\mathrm{TTCTT})_{3}$ & / & - & - \\
\hline LaES26 & $(\mathrm{TAT})_{5}$ & $(\mathrm{TAT})_{5}$ & $(\mathrm{TAT})_{5}$ & / & / & / \\
\hline LaES27 & $(\mathrm{TGG})_{5}$ & $(\mathrm{TGG})_{5}$ & $(\mathrm{TGG})_{4}$ & I & / & / \\
\hline LaES29 & $(A A G A)_{5}$ & $(\mathrm{AAGA})_{5}$ & / & / & / & / \\
\hline LaES31 & $(\mathrm{CTC})_{5}$ & $(\mathrm{CTC})_{7}$ & / & / & - & / \\
\hline LaES34 & $(\mathrm{GTT})_{5}$ & $(\mathrm{GTT})_{5}$ & $(\mathrm{GTT})_{4}$ & / & - & / \\
\hline LaES36 & $(\mathrm{CT})_{8}$ & $(\mathrm{CT})_{7}$ & $(\mathrm{CT})_{7}$ & $(\mathrm{CT})_{6}$ & $(\mathrm{CT})_{7}$ & $(\mathrm{CT})_{8}$ \\
\hline LaES41 & $(G A G)_{6}$ & $(\mathrm{GAG})_{5}$ & / & / & - & - \\
\hline LaES46 & $(\mathrm{AGTGC})_{4}$ & $(\mathrm{AGTGC})_{4}$ & / & / & / & / \\
\hline LaES49 & $(T A)_{7}$ & $(T A)_{7}$ & $(\mathrm{TA})_{5}$ & I & - & - \\
\hline LaES53 & $(\mathrm{CAT})_{6}$ & $(\mathrm{CAT})_{7}$ & $(\mathrm{CAT})_{8}$ & $(\mathrm{CAT})_{8}$ & $(\mathrm{CAT})_{5}$ & $(\mathrm{CAT})_{5}$ \\
\hline LaES55 & $(\mathrm{AGC})_{5}$ & $(\mathrm{AGC})_{5}$ & / & I & - & - \\
\hline LaES58 & $(\mathrm{ACC})_{5}$ & $(\mathrm{ACC})_{5}$ & / & I & - & I \\
\hline
\end{tabular}

Note: ' $/$ ' indicated that products were not sequenced, and ' - ' indicates no products or products with improper sizes

suggested that the SSR primers with proper size products are probably authentic SSRs, further denoting high potential of the set of EST-SSRs.

\section{GO (gene ontology) annotation of genes harboring the set of SSRs}

EST-SSRs are derived from transcribed genes and hence the annotation of the corresponding genes will be helpful for their application in trait associated marker selection [32]. GO annotations of the genes harboring ESTSSRs are listed in Table S1 according to the annotations of Wang et al. [27]. Among 839 candidate EST-SSRs, 373 have GO annotations. Of the 60 EST-SSRs, 52 have GO annotations, among which 16 EST-SSRs (LaES1, LaES19, LaES23, LaES26, LaES29, LaES31, LaES32, LaES33, LaES34, LaES35, LaES42, LaES43, LaES46, LaES47, LaES50, LaES58) are assigned to signaling or stimulus responses, 14 (LaES3, LaES16, LaES17, LaES18, LaES20, LaES22, LaES36, LaES44, LaES48, LaES51, LaES55, LaES56, LaES57, LaES59) are associated with the development of floral organs, three (LaES25, LaES49, LaES53) are involved in vegetative phase change and the remaining 19 participate in processes such as Golgi organization and cysteine biosynthesis. As the transcriptome sequences were obtained from seedlings of $L$. aurea treated with MJ, it is acceptable that many of the genes were associated with processes involved in responses to stimulus or signals.

\section{Genetic diversity and population structure analysis of $L$. radiata lines}

To apply the newly developed SSR markers for genetic analysis, $7 \mathrm{~L}$. radiata lines with difference in fertility, were selected for diversity analysis (Table 4). Of these, three are fertile, three are sterile, and one line consisted of the progeny of one fertile line, Pop6. Considering the GO annotations and transferability of the set of 60 ESTSSRs, 15 EST-SSR markers, containing 10 sequenceverified and 5 unverified SSR markers (LaES3, LaES20, LaES31, LaES46 and LaES58) were chosen to explore the genetic diversity of the $7 \mathrm{~L}$. radiata lines.

A total of 88 bands were detected in the L. radiata lines, among which 80 were polymorphic. The parameters of genetic diversity and differentiation were described in Tables 5 and 6, respectively. Polymorphism information content (PIC) values of the used EST-SSR markers ranged from 0.374 to 0.850 , except for LaES18 and LaES27, which demonstrated no polymorphism in the $L$. radiata lines. The mean PIC value among the verified and unverified markers were 0.636 and 0.681 , 
Table 3 Cross-species amplification of the 60 microsatellite loci in Lycoris species

\begin{tabular}{|c|c|c|c|c|c|}
\hline \multirow[t]{2}{*}{ Markers } & \multicolumn{5}{|c|}{ Amplification } \\
\hline & in L. aurea & in L. chinensis & in L. anhuiensis & in L. radiata & in L. sprenger \\
\hline LaES1 & + & + & + & + & + \\
\hline LaES2 & - & - & - & - & - \\
\hline LaES3 & + & + & + & + & + \\
\hline LaES4 & + & + & + & + & + \\
\hline LaES5 & + & + & + & + & + \\
\hline LaES6 & + & + & + & + & + \\
\hline LaES7 & + & + & + & + & + \\
\hline LaES8 & - & - & - & - & - \\
\hline LaES9 & - & - & - & - & - \\
\hline LaES10 & + & + & + & + & + \\
\hline LaES11 & - & - & - & - & - \\
\hline LaES12 & + & + & + & + & + \\
\hline LaES13 & + & + & + & + & + \\
\hline LaES14 & + & + & + & + & + \\
\hline LaES15 & + & - & + & + & + \\
\hline LaES16 & - & - & - & - & - \\
\hline LaES17 & - & - & - & - & - \\
\hline LaES18 & + & + & + & + & + \\
\hline LaES19 & + & - & - & - & - \\
\hline LaES20 & + & - & + & + & + \\
\hline LaES21 & + & - & - & + & + \\
\hline LaES22 & + & + & + & + & + \\
\hline LaES23 & - & - & - & + & + \\
\hline LaES24 & - & - & - & - & + \\
\hline LaES25 & + & - & - & + & + \\
\hline LaES26 & + & + & + & + & + \\
\hline LaES27 & + & + & + & + & + \\
\hline LaES28 & + & - & + & + & + \\
\hline LaES29 & + & + & + & + & + \\
\hline LaES30 & + & - & - & + & + \\
\hline LaES31 & + & - & + & + & + \\
\hline LaES32 & + & - & - & + & + \\
\hline LaES33 & + & - & - & + & + \\
\hline LaES34 & + & + & + & + & + \\
\hline LaES35 & - & - & - & - & - \\
\hline LaES36 & + & + & + & + & + \\
\hline LaES37 & - & - & - & - & - \\
\hline LaES38 & - & - & - & - & - \\
\hline LaES39 & + & - & - & + & + \\
\hline LaES40 & + & - & - & + & + \\
\hline LaES41 & + & - & - & + & + \\
\hline LaES42 & + & - & - & + & + \\
\hline LaES43 & + & - & - & + & + \\
\hline LaES44 & - & - & - & - & - \\
\hline LaES45 & - & - & - & - & - \\
\hline
\end{tabular}


Table 3 Cross-species amplification of the 60 microsatellite loci in Lycoris species (Continued)

\begin{tabular}{|c|c|c|c|c|c|}
\hline \multirow[t]{2}{*}{ Markers } & \multicolumn{5}{|c|}{ Amplification } \\
\hline & in L. aurea & in L. chinensis & in $L$. anhuiensis & in L. radiata & in L. sprengeri \\
\hline LaES46 & + & + & + & + & + \\
\hline LaES47 & + & + & + & + & + \\
\hline LaES48 & + & - & + & + & + \\
\hline LaES49 & + & - & - & + & + \\
\hline LaES50 & - & - & - & - & - \\
\hline LaES51 & + & - & + & + & + \\
\hline LaES52 & + & + & + & + & + \\
\hline LaES53 & + & + & + & + & + \\
\hline LaES54 & - & - & - & - & - \\
\hline LaES55 & + & - & - & + & + \\
\hline LaES56 & + & - & - & + & + \\
\hline LaES57 & - & - & - & - & - \\
\hline LaES58 & + & - & + & + & + \\
\hline LaES59 & + & - & + & + & + \\
\hline LaES60 & + & + & + & + & + \\
\hline Total & 44 & 23 & 30 & 44 & 45 \\
\hline
\end{tabular}

Note: ' + ' indicates that products with proper sizes, '-' indicates no products or products with improper sizes

respectively (Table 5). Also, the genetic differentiation (Gst) and the gene flow $(\mathrm{Nm})$ parameters were similar between the verified and the unverified markers (Table 6), which suggested that both SSR groups are useful for genetic analysis in L. radiata.

The average genetic differentiation parameter Gst was 0.752 among the $7 \mathrm{~L}$. radiata lines, which means that diversity between populations accounted for $75.2 \%$ of total diversity, suggesting high genetic diversity among populations. Nevertheless, genetic communication among those $L$. radiata lines was very low, for $\mathrm{Nm}$ was just 0.172 (Table 6). We propose that the low genetic communication among $L$ radiata lines may result from asexual reproduction, which is the main reproduction mode of Lycoris. Therefore, genetic diversity assessment of Lycoris accessions collected from different habitats is important and valuable for the development of elite germplasm.

In order to elucidate the genetic relationship of these $7 L$. radiata lines, $L$. aurea was added as an outgroup to construct a dendrogram based on Nei's genetic distance. As shown in Table 7, Nei's genetic distances presented high variance between these lines, ranging from 0.0064 to 0.5568 . The distance between $L$. aurea and Pop4 was the furthest, while that between Pop2 and Pop3 was the closest, then that between Pop6 and Pop7. Correspondingly, a dendrogram was constructed based on the genetic distance using the unweighted pair group method with arithmetic mean analysis (UPGMA). As shown in Fig. 2, the outgroup was separated from $L$. radiata, while $L$. radiata lines were clustered into two groups. Interestingly, these two groups were consistent with their fertility. The three sterile lines comprised group I, while the three fertile lines plus the offspring population formed group II. These results hinted high efficiency of the 15 EST-SSRs.

To further evaluate the relationship of the $34 L$. radiata individuals, population structure was analyzed with Structure 2.3.4. The $\Delta \mathrm{K}$ method showed that the optimal $\mathrm{K}$ value was $\mathrm{K}=4$ (Fig. 3a), which inferred the existence of four main groups among the $34 \mathrm{~L}$. radiata individuals. As shown in Fig. 3b, individuals from each line had the same ancestry except for lines 6 and 7 , among which some individuals were scattered in more than one group, suggesting admixed ancestry of Pop6 and Pop7. From the Q value profile of populations (Table S3), it was also concluded that Pop6 and Pop7 were admixed. As shown in Fig. 3b, 6 individuals containing one from Pop6, the parent line, and five from offspring Pop7 were assigned to intermediate. It is acceptable that a parental line with an admixed ancestry would produce greater diversity in its offspring line.

\section{Discussion}

Lycoris species laid a little behind other species in genetic research. So far, some molecular markers such as SCoT, ITS, have been used to determine the genetic diversity and evolutionary relationship among Lycoris species [33, 34]. However, these molecular markers are not sufficient for such studies as linkage group construction and marker assisted selection (MAS). Instead, SSRs are powerful DNA markers for this type of research, because of their co-dominant inheritance. Though there 

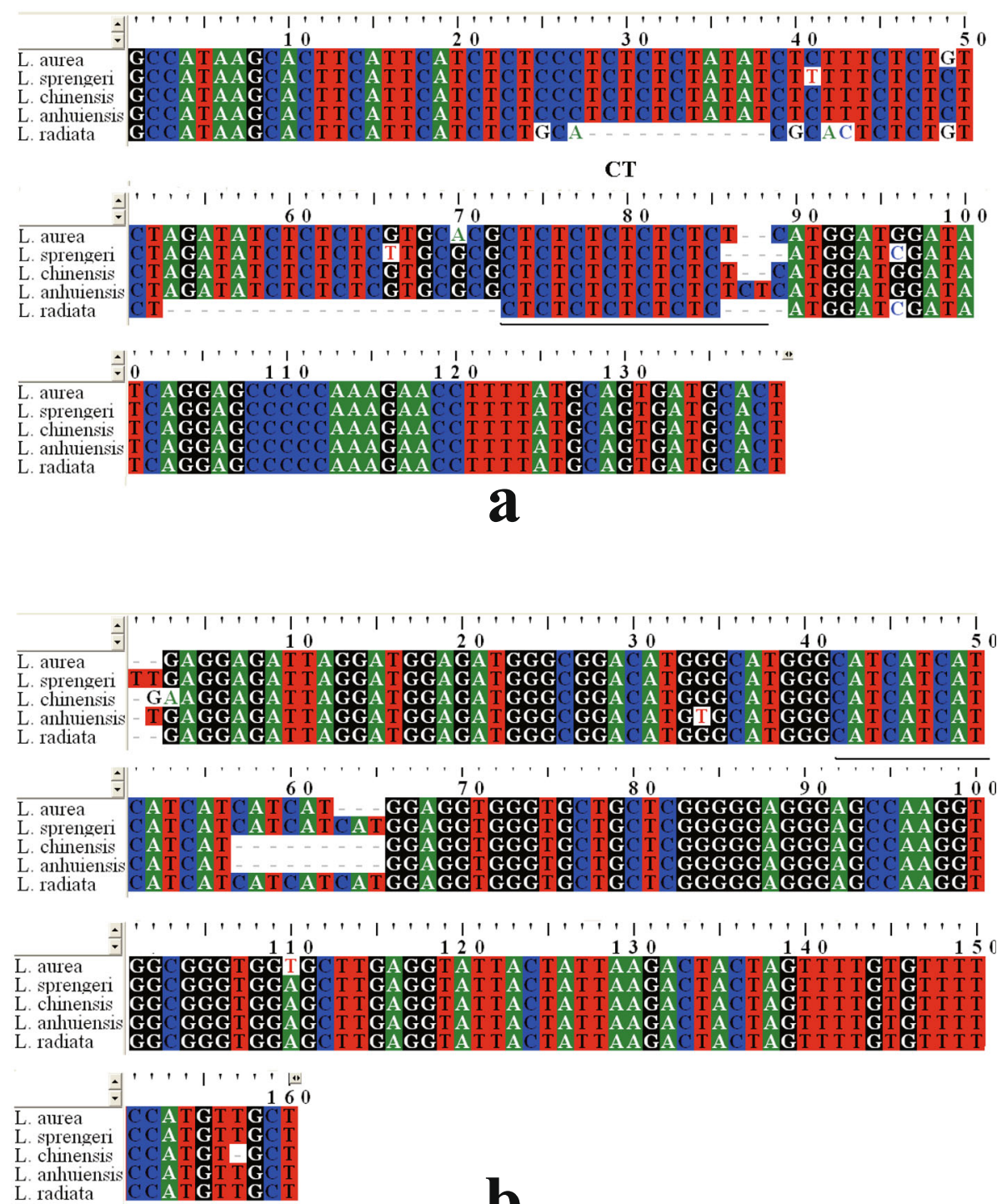

Fig. 1 Alignment of amplicons of LaES36 and LaES53 in L. aurea, L. sprengeri, L. chinensis, L. anhuiensis and L. radiata. The repeat motifs are underlined. a, Alignment for LaES36. b. Alignment for LaES53

have been 27 genomic SSR markers and 27 genic SSR markers available in Lycoris species [25, 29-31], they are far from adequate for better genetic analysis of Lycoris genus. Since the Lycoris genus has a massive genome [15], a large number of DNA markers are necessary to cover the whole genome. Thus, the list of 839 potential EST-SSR markers released in this study will be helpful for genetic analysis, especially for genetic improvement of Lycoris.

Transcriptome sequencing technology makes it easier to develop EST-SSRs, and nowadays abundant EST-SSRs are available [35-37]. However, sequence redundancy is a major disadvantage of EST-SSRs due to multiple alternative transcripts for the same gene [21]. In addition, some SSRs occasionally exist in reverse complementary formats, but in fact they are identical. However, these common phenomenon about EST-SSRs from transcriptome sequences were often ignored in the development of EST-SSRs. Thus, the actual number of EST-SSRs obtained from transcriptome sequences should be much less than that detected. Moreover, such redundancy will reduce the representativeness of the EST-SSRs developed, and then their efficiency in application. Bazzo et al. successfully developed a total of 418 SSRs from 7492 detected EST-SSRs after considering their redundancy from transcriptome sequences of macaúba palm (Acrocomia aculeata) [37]. In our study, thousands of EST-SSRs were detected from transcriptome sequences 
Table 4 L. radiata lines used for genetic diversity analysis

\begin{tabular}{|c|c|c|c|c|c|}
\hline Population Code & Individual Numbers & Karotype & Ploidy & Characters & Places of Collection \\
\hline Pop1 & 3 & $2 n=33$ & triploid & sterile & $\begin{array}{l}\text { Jiuhua, Jurong, Jiangsu } \\
\left(119^{\circ} 13^{\prime} \mathrm{E}, 32^{\circ} 07^{\prime} \mathrm{N}\right)\end{array}$ \\
\hline Pop2 & 3 & $2 n=22$ & diploid & sterile & $\begin{array}{l}\text { Nanjing Botanical Garden Mem. Sun Yat-Sen } \\
\left(118^{\circ} 83^{\prime} E, 32^{\circ} 05^{\prime} N\right)\end{array}$ \\
\hline Pop3 & 4 & $2 n=22$ & diploid & sterile & $\begin{array}{l}\text { Nanjing Botanical Garden Mem. Sun Yat-Sen } \\
\left(118^{\circ} 83^{\prime} \mathrm{E}, 32^{\circ} 05^{\prime} \mathrm{N}\right)\end{array}$ \\
\hline Pop4 & 2 & $2 n=22$ & diploid & fertile & $\begin{array}{l}\text { Yixing, Wuxi, Jiangsu } \\
\left(119^{\circ} 82^{\prime} \mathrm{E}, 31^{\circ} 34^{\prime} \mathrm{N}\right)\end{array}$ \\
\hline Pop5 & 3 & $2 n=22$ & diploid & fertile & $\begin{array}{l}\text { Pengze, Jiujiang, Jiangxi } \\
\left(116^{\circ} 56^{\prime} E, 29^{\circ} 09^{\prime} N\right)\end{array}$ \\
\hline Pop6 & 4 & $2 n=22$ & diploid & fertile & $\begin{array}{l}\text { Suxian,Chenzhou, Hunan } \\
\left(113^{\circ} 06^{\prime} \mathrm{E}, 25^{\circ} 55^{\prime} \mathrm{N}\right)\end{array}$ \\
\hline Pop7 & 15 & $2 n=22$ & diploid & offspring & $\begin{array}{l}\text { Offspring of Pop6 } \\
\left(118^{\circ} 83^{\prime} \mathrm{E}, 32^{\circ} 05^{\prime} \mathrm{N}\right)\end{array}$ \\
\hline
\end{tabular}

of $L$. aurea seedlings treated with $\mathrm{MJ}$, but many of these EST-SSRs were redundant duplicates. By eliminating duplicated EST-SSRs, we identified 839 SSRs from 4637 detected EST-SSRs as a pool of non-redundant candidate EST-SSRs, which would be promising for SSR development and correspondingly for genetic analysis.

When EST-SSRs are developed from transcribed eukaryotic genes, introns may affect the amplification products. Additionally, assembly errors may also affect amplification of the SSRs $[35,36]$. Therefore, in this study, sequencing confirmation was conducted in the

Table 5 Parameters of genetic diversity among 15 EST-SSR markers for $7 \mathrm{~L}$. radiata lines

\begin{tabular}{lllllll}
\hline Markers & & Na & Ne & h & I & PIC \\
\hline Sequence verified & LaES13 & 16 & 11.549 & 0.275 & 0.428 & 0.817 \\
& LaES18 & 1 & 1 & 0 & 0 & 0 \\
& LaES22 & 3 & 2.371 & 0.135 & 0.221 & 0.374 \\
& LaES25 & 20 & 13.195 & 0.209 & 0.339 & 0.765 \\
& LaES26 & 7 & 5.098 & 0.155 & 0.244 & 0.447 \\
& LaES27 & 1 & 1 & 0 & 0 & 0 \\
LaES34 & 9 & 7.181 & 0.238 & 0.354 & 0.615 \\
Mean & LaES36 & 22 & 16.280 & 0.299 & 0.462 & 0.850 \\
& LaES49 & 6 & 4.868 & 0.371 & 0.554 & 0.474 \\
& LaES53 & 16 & 11.374 & 0.236 & 0.357 & 0.742 \\
& 10.1 & 7.391 & 0.192 & 0.296 & 0.636 \\
& LaES03 & 12 & 8.181 & 0.254 & 0.415 & 0.708 \\
& LaES20 & 18 & 13.527 & 0.300 & 0.455 & 0.828 \\
& LaES31 & 17 & 12.168 & 0.373 & 0.390 & 0.801 \\
& LaES46 & 7 & 4.812 & 0.153 & 0.262 & 0.603 \\
& LaES58 & 13 & 8.451 & 0.130 & 0.217 & 0.466 \\
& 13.4 & 9.428 & 0.242 & 0.348 & 0.681 \\
\hline
\end{tabular}

$\mathrm{Na}$ Observed number of alleles, Ne Effective number of alleles, $h$ Nei's (1973) gene diversity, I Shannon's Information index, PIC Polymorphism information content

amplicons that generated clear bands of expected sizes, and the results confirmed that the designed primers amplified the expected loci. Supposing the same amplification rate of the subset of EST-SSRs in L. aurea, there would be about 615 primer pairs in the primer pool which can be developed as EST-SSR markers for $L$. aurea, suggesting great potential of the primer pool.

EST-SSRs have a high transferability among closely related species $[35,36,38,39]$. When assaying the transferability of EST-SSRs, amplification condition affecting the effectiveness and fidelity of amplification is an important factor [19]. In this study, the annealing temperature was set at $58^{\circ} \mathrm{C}$ to avoid unspecific amplification when performing PCR amplification in all the five

Table 6 Parameters of genetic differentiation among 13 ESTSSR markers for $7 \mathrm{~L}$. radiata lines

\begin{tabular}{llllll}
\hline Markers & & Ht & Hs & Gst & Nm \\
\hline Verified & LaES13 & 0.304 & 0.033 & 0.891 & 0.061 \\
& LaES22 & 0.168 & 0.036 & 0.786 & 0.136 \\
& LaES25 & 0.202 & 0.054 & 0.733 & 0.182 \\
& LaES26 & 0.133 & 0.046 & 0.654 & 0.264 \\
& LaES34 & 0.248 & 0.055 & 0.778 & 0.142 \\
& LaES36 & 0.300 & 0.061 & 0.797 & 0.128 \\
Mean & LaES49 & 0.432 & 0.060 & 0.861 & 0.081 \\
Unverified & LaES53 & 0.220 & 0.059 & 0.732 & 0.183 \\
& LaES03 & 0.260 & 0.040 & 0.779 & 0.147 \\
& LaES20 & 0.325 & 0.052 & 0.840 & 0.095 \\
& LaES31 & 0.258 & 0.102 & 0.605 & 0.327 \\
Mean & LaES46 & 0.200 & 0.058 & 0.710 & 0.204 \\
differentiation coefficient, $N m$ Gene flow) & & 0.702 & 0.212 \\
& LaES58 & 0.106 & 0.033 & 0.689 & 0.226 \\
& & 0.230 & 0.065 & 0.709 & 0.213 \\
\hline
\end{tabular}


Table 7 Pairwise comparison of Nei's genetic distance among populations

\begin{tabular}{|c|c|c|c|c|c|c|c|c|c|}
\hline \multirow[t]{2}{*}{ Pop ID } & & \multirow{2}{*}{$\begin{array}{l}\text { L. } \\
\text { aurea }\end{array}$} & \multicolumn{7}{|c|}{ L. radiata } \\
\hline & & & Pop1 & Pop2 & Pop3 & Pop4 & Pop5 & Pop6 & Pop7 \\
\hline \multicolumn{10}{|l|}{ L. aurea } \\
\hline \multirow[t]{7}{*}{ L. radiata } & Pop1 & 0.3988 & & & & & & & \\
\hline & Pop2 & 0.5364 & 0.2666 & & & & & & \\
\hline & Pop3 & 0.5316 & 0.2499 & 0.0064 & & & & & \\
\hline & Pop4 & 0.5568 & 0.4424 & 0.4327 & 0.4105 & & & & \\
\hline & Pop5 & 0.5125 & 0.3483 & 0.3401 & 0.3219 & 0.2246 & & & \\
\hline & Pop6 & 0.3352 & 0.2529 & 0.2631 & 0.2397 & 0.2362 & 0.1615 & & \\
\hline & Pop7 & 0.3121 & 0.2197 & 0.2284 & 0.2085 & 0.2034 & 0.1392 & 0.0304 & \\
\hline
\end{tabular}

species. In total, 23 out of 44 EST-SSRs from L. aurea were transferable among $L$. radiata, L. sprengeri, $L$. chinensis and L. anhuiensis, which accounted for $52.27 \%$ transferability, higher than that observed with genomic SSRs [30, 31]. Interestingly, it was found that the transferability was high in $L$. radiata and $L$. sprengeri, whereas it was lower in L. chinensis and L. anhuiensis. It is known that L. aurea, L. chinensis, L. anhuiensis, $L$. radiata and $L$. sprengeri are common and primitive species of Lycoris and also that some Lycoris species that originated from hybridization are mostly hybrids derived from the above species $[14,15]$. Thus, EST-SSR transferability observed in this study will supply informative and practical guidance for application of this set of ESTSSRs in Lycoris.

The polymorphism information content (PIC) is an important parameter of a DNA marker to reflect the power of the molecular marker. In general, markers with PIC> 0.5 are defined as highly polymorphic [40]. In our case, the average PIC value of the 13 EST-SSR markers used, except LaES18 and LaES27, was 0.654, higher than many EST-SSR markers developed from transcriptome sequences, such as for Torreya grandis (0.357) [41], Mucuna pruriens (0.24) [42] and Lagerstroemia spp (0.589) [43]. The high PIC values observed in our work hinted a high efficiency of these set of markers in genetic analysis.

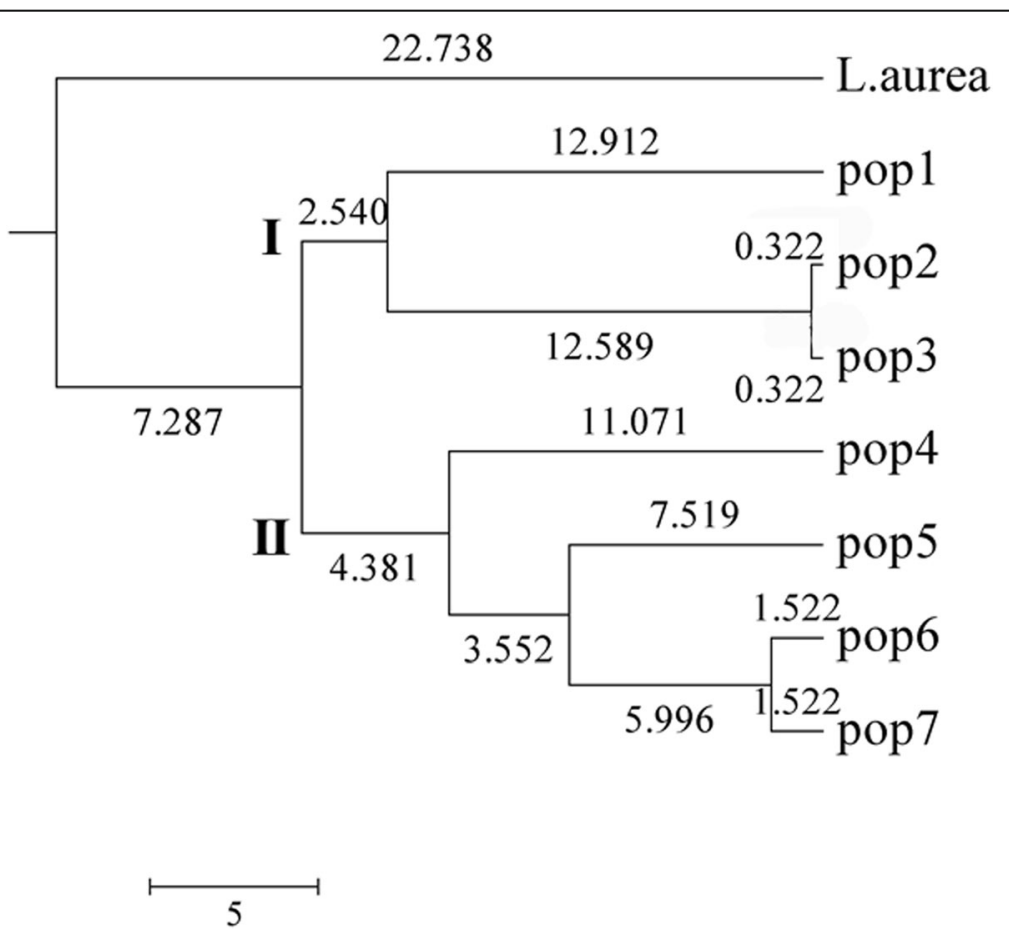

Fig. 2 UPGMA dendrogram constructed among 34 individuals from 7 L. radiata lines with L. aurea as an outgroup based on 15 SSR markers developed in this study 

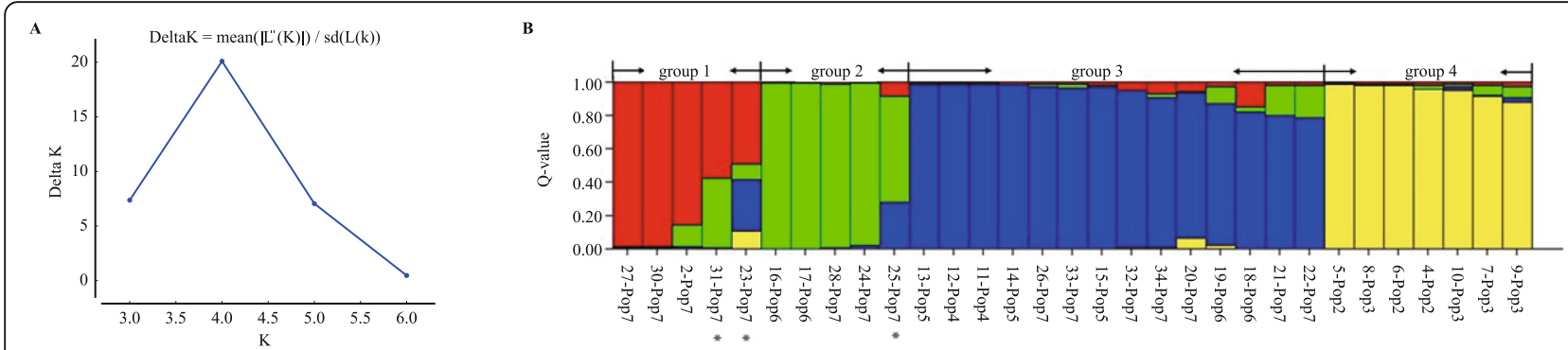

Fig. 3 Structure analysis of L. radiata lines. a Plot of Delta K vs K values to define the most probable number of clusters in the analysis of population structure of 34 Lycoris radiata individuals; $\mathbf{b} Q$ values and ancestry assignment of Lycoris radiata individuals $(Q>0.70, K=4)$. Admixed or intermediate were identified with an asterisk. The Arabic numerals represented individuals

The genetic relationship of the $7 \mathrm{~L}$. radiata lines and L. aurea was analyzed by using the 15 EST-SSR markers. The results showed a significant genetic distance between $L$. aurea and the $L$. radiata lines, with an average of 0.4547. The closest genetic distance, 0.0064, was found between Pop2 and Pop3, two lines from Nanjing Botanical Garden Mem. Sun Yat-Sen, which are possibly the same accessions. The Q value profile of the Structure analysis also indicated the same ancestry for Pop2 and Pop3 (Table S3). The genetic distance between an offspring line, Pop7, and its parent line, Pop6, was 0.0304 , also suggesting a close relationship. In addition, the $Q$ value profile demonstrated that both Pop6 and Pop7 had an admixed ancestry of groups 1, 2, and 3, which is reasonable for an admixed parent line can yield an admixed offspring. In general, the genetic distances and genetic structure based on the mere 15 EST-SSRs were reasonable and acceptable.

Because EST-SSR markers are identified from transcribed RNA sequences and may be linked to functional genes with a possible impact on important traits, ESTSSRs may have advantages over genomic SSRs [19]. For example, González et al. focused on EST-SSRs from genes involved in some specific pathways, and three EST-SSRs were even able to discriminate different properties of the fruits [32]. In our case, the dendrogram based on mere 15 EST-SSRs showed that the outgroup, $L$. aurea was separated from $L$. radiata lines and that the $7 \mathrm{~L}$. radiata lines were clustered into two groups consistent with their fertility. Moreover, the UPGMA dendrogram of the $34 \mathrm{~L}$. radiata individuals based on 15 EST-SSR markers clustered all the individuals into two groups as well (Fig. 4), similar to that obtained from the analysis with the $7 \mathrm{~L}$. radiata lines and an outgroup (Fig. 2). The first group consisted of all the individuals from three sterile lines, plus two individuals from Pop7, and another group was comprised of the remaining individuals from the fertile lines. Considering that LaES18 and LaES27 showed no polymorphism in $L$. radiata lines, the dendrogram was just based on the 13 EST-SSR markers, suggesting that those 13 EST-SSR markers are highly efficient. And the high efficiency may be associated with the functions of those transcribed genes. Although the mechanisms of fertility in L. radiata is unclear, many studies demonstrated that gibberellic acid (GA) and auxin signaling are involved in regulating reproductive development $[44,45]$. Since crosstalk among ethylene, ABA, GA and auxin is undoubted [46], all these signals may play roles in reproductive development. Additionally, we speculated that processes of flower organ development as well as vegetative phase change, may affect seed development and finally result in a productive trait: fertility or sterility. Interestingly, among the 13 EST-SSRs, 4 are involved in hormonal signal response, 4 are associated with flower organ development and 3 participate in vegetative phase change. Furthermore, correlation analysis showed that 28 loci (LaES36-3, - 4, - 5, - 7, - 10, - 11, LaES49-2, LaES532, - 3, - 8, LaES58-1, LaES3-1, - 4, LaES25-4, - 5, - 7, - 8, LaES34-4, S31-4, - 5, - 6, LaES13-2, - 3, - 4, - 7, LaES20-1, - 2, -6) from the 13 EST-SSR markers were significantly correlated with fertility (Table S5). Actually, these loci were from 10 of the above 11 EST-SSR markers, associated with flower organ development, vegetative phase change and hormonal signal response. In general, GO annotations of these 10 EST-SSRs may explain the high consistency between the clusters and fertility. Nonetheless, more work and efforts are needed to elucidate the mechanism of fertility in L. radiata.

\section{Conclusions}

This study included the mining of EST-SSR markers from transcriptome data of $L$. aurea. A potential pool of 839 non-redundant EST-SSR markers was supplied for Lycoris. Marker characterization and validation demonstrated that the newly developed SSR markers have high amplification rate and transferability. Moreover, nearly half of the set of SSRs have GO annotations, which would be useful for trait associated marker selection in Lycoris. Further, 15 EST-SSRs were selected for the genetic diversity analysis of $7 \mathrm{~L}$. radiata lines, consequently the $7 \mathrm{~L}$. radiata lines were clustered into two groups 


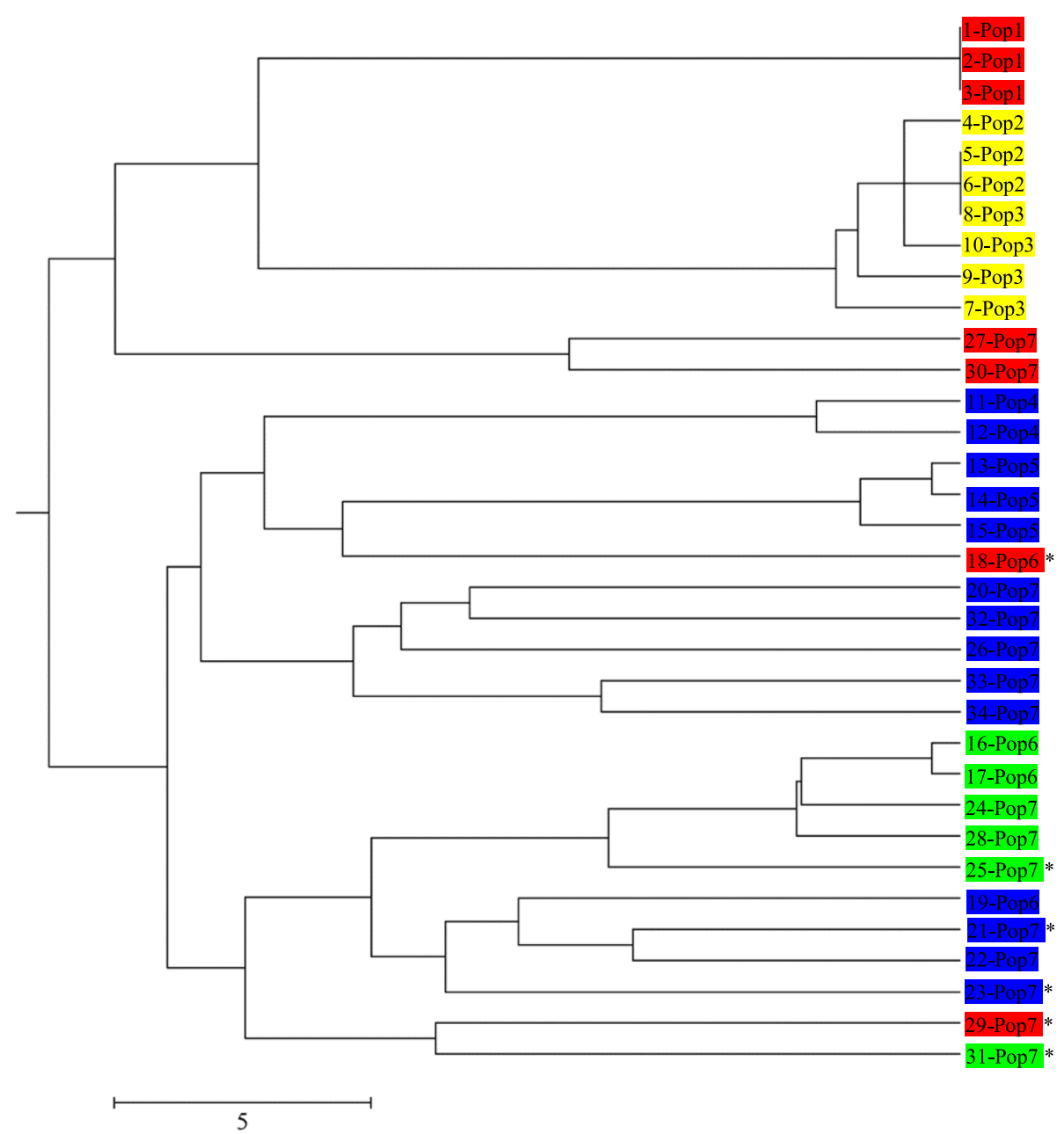

Fig. 4 UPGMA dendrogram of the 34 L. radiata individuals based on 15 SSR markers. Admixed or intermediate were identified with an asterisk. Color was set consistent with structure analysis. The Arabic numerals represented individuals

consistent with fertility, suggesting a high efficiency of the EST-SSRs. The set of EST-SSRs would facilitate diversity analysis and breeding in Lycoris genus.

\section{Methods}

\section{Plant materials}

The plant materials include $11 \mathrm{~L}$. aurea individuals and 34 individuals of $L$. radiata, and one individual of each L. sprengeri, L. chinensis and L. anhuiensis. Individuals of L. aurea, L. sprengeri, L. chinensis and $L$. anhuiensis were collected from Nanjing Botanical Garden Mem. Sun Yat-Sen $\left(118^{\circ} 83^{\prime} \mathrm{E}, 32^{\circ} 05^{\prime} \mathrm{N}\right)$. Collection information and characteristics of $L$. radiata lines are provided in Table 4 . The voucher specimens were identified by Prof. Feng Peng and deposited in the Herbarium of Institute of Botany, Jiangsu Province and Chinese Academy of Sciences. The plant materials used in this study were cultivated in the nursery of Nanjing Botanical Garden Mem. Sun Yat-Sen, Nanjing, China. Seeds of one line (Pop6) were collected from the nursery in November of 2017 and germinated in an illuminated incubator at $22^{\circ} \mathrm{C}, 2000 \mathrm{~lx}$, and a $16 \mathrm{~h} / 8 \mathrm{~h} \mathrm{light} /$ dark (L/D) cycle. Young leaves of the above individuals were collected for DNA extraction.

EST-SSR validation was performed in $L$. aurea (Table S2). Cross-species transferability analysis of EST-SSRs was performed in $L$. radiata, $L$. sprengeri, $L$. chinensis and $L$. anhuiensis. Thirty-four $L$. radiata individuals that belong to 7 lines were used for genetic diversity analysis (Table 4).

\section{SSR mining and primer design}

The MISA [47] was used for microsatellites screening. Perfect di-, tri-, tetra-, penta-, and hexa-nucleotide motifs were detected by setting the parameters to a minimum of $6,5,4,4$, and 4 repeats, respectively. SSR sequences in different transcripts of the same gene were aligned using BioEdit to detect duplicate EST-SSRs. Primer pairs were designed using software Primer3 (http://bioinfo.ut.ee/pri mer3/) with the parameters set as: primer length of 18-24 
bases, GC content of $40-60 \%$, annealing temperatures of $52-60^{\circ} \mathrm{C}$, and PCR product size of $80-300 \mathrm{bp}$.

\section{DNA extraction}

Young leaves of each individual were ground into powder with liquid nitrogen. DNA extraction was carried out according to instructions of the plant genomic DNA Mini Kit (Tiangen, Beijing, China), and DNA was detected on $1 \%$ agarose gel to evaluate DNA quality and concentration. The total DNA samples were diluted at concentration of $20 \mathrm{ng} / \mu \mathrm{L}$ with TE buffer and stored at $-20^{\circ} \mathrm{C}$ for PCR amplification.

\section{EST-SSR validation}

PCR reactions were carried out in a $20 \mu \mathrm{L}$ reaction volume, containing $20 \mathrm{ng}$ of genomic DNA, $0.5 \mu \mathrm{M}$ of each primer, and $10 \mu \mathrm{L}$ of $2 \mathrm{x}$ Taq Master Mix (Dye Plus) (Vazyme Biotech, Nanjing, China). The PCR reactions were performed in an Eppendorf Mastercycler ep gradient thermal cycler using the following program: $3 \mathrm{~min}$ at $94{ }^{\circ} \mathrm{C}$, followed by 35 cycles of $30 \mathrm{~s}$ at $94^{\circ} \mathrm{C}, 30 \mathrm{~s}$ at $58^{\circ} \mathrm{C}$, and $30 \mathrm{~s}$ at $72{ }^{\circ} \mathrm{C}$, then a final extension at $72{ }^{\circ} \mathrm{C}$ for 5 min. PCR products were analyzed via $2 \%$ MetaPhor $^{\text {mix }}$ agarose (Lonza.com) gel electrophoresis, and products with strong and clear band were cloned into a T-vector and sequenced.

\section{Genetic diversity analysis in L. radiata}

PCR products were separated on $8 \%$ non-denaturing polyacrylamide gels for polymorphism analysis and visualized by silver staining. PCR products were manually scored based on allele size following data scoring as "0" in the absence of the band and " 1 " as its presence. The binary data matrix was subjected to POPGENE1.32. Population genetic parameters of $7 \mathrm{~L}$. radiata lines, $(\mathrm{Na}$, $\mathrm{Ne}, \mathrm{h}, \mathrm{I})$ and differentiation parameters (Ht, Hs, Gst and $\mathrm{Nm}$ ) were evaluated by POPGENE version 1.32 [48] and PIC by PIC_CALC [49]. The UPGMA dendrogram of lines was constructed with $L$. aurea as an outgroup based on the genetic similarities, allowing a 1000 replicate bootstrap. Similarly, an UPGMA dendrogram was constructed with the $34 \mathrm{~L}$. radiata individuals.

Statistical analyses were conducted using SPSS statistics (version 20), applying the Spearman correlation coefficient test.

\section{Population structure analysis}

An analysis of population structure and ancestry of the $34 \mathrm{~L}$. radiata individuals based on Bayesian statistics, without prior assignment to populations, was performed using Structure v.2.3.4 [50, 51]. In this study, SSRs were applied as dominant markers and the binary data $(0,1)$ were used. Since the input data in different ploidy models is not acceptable in Structure 2.3.4, Pop1, a triploid population was denoted as a diploid population. Batch runs with correlated and independent allele frequencies among inferred clusters were tested with population parameters set to admixture model (burn-in 50,000; run-length 100,000). The program Structure Harvester (http:// taylor0.biology. ucla.edu/structure Harvester/\#) was used to estimate the final $\mathrm{K}$ value for the STRUCTURE analysis based on both the Plot of mean posterior probability $(\mathrm{LnP}(\mathrm{D}))$ values and the ad hoc Evanno's $\Delta \mathrm{K}$ statistics [52]. $L$. radiata individuals were allocated to a cluster if $\mathrm{Q}$ values were greater or equal to 0.70 , or otherwise considered as intermediate or admixed.

\section{Supplementary Information}

The online version contains supplementary material available at https://doi. org/10.1186/s12870-020-02727-3.

Additional file 1: Table S1. Contig sequence and primer information of 839 microsatellite markers developed for Lycoris.

Additional file 2: Table S2. Collection information of $11 \mathrm{~L}$. aurea individuals.

Additional file 3: Table S3. Q value profile of the $7 \mathrm{~L}$. radiata lines. Additional file 4: Table S4. Q value profile of the $34 \mathrm{~L}$. radiata individuals.

Additional file 5: Table S5. Correlation coefficients among fertility and loci.

Additional file 6: Table S6. Sequences of EST-SSRs validated in Lycoris species.

\section{Abbreviations}

SSR: Simple sequence repeats; ESTs: Expressed sequence tags; ESTSSR: Simple sequence repeats in expressed sequence tags; UPGMA: The unweighted pair group method with arithmetic means; MJ: Methyl jasmonate; MAS: Marker assisted selection; GO: Gene ontology;

GA: Gibberellic acid; SCOT: Start codon targeted marker; Na: Observed number of alleles; Ne: Effective number of alleles; h: Nei's (1973) gene diversity; I: Shannon's Information index; Ht: Total genetic diversity; Hs: Within population genetic diversity; Gst: Genetic differentiation coefficient; PIC: Polymorphism information content; Nm: Gene flow; Pop: Population; L/ D: Light/dark

\section{Acknowledgements}

We would like to thank Dr. Guoqi Yao, Maize Research Institute, Shandong Academy of Agricultural Sciences for academic help.

\section{Authors' contributions}

RW designed the research; YMJ, SX, RW performed most of the experiments and data analysis; SX, RW performed data mining analysis; JYZ performed statistical genetic analyses; YMJ developed and screened the EST-SSR markers and wrote the draft of the paper. JD, and QY performed the investigation and participated in sample collection. All authors have read and approved the final manuscript

\section{Funding}

This research was financially supported by National Natural Science Foundation of China (Grant Nos. 81603240, 31770387), High-level talents project of Six Talent Peaks in Jiangsu Province (Grant No. NY-081) and "the 333" project of Jiangsu Province (Grant No. BRA2020082). The funders were not involved in the experiment design, data collection and analysis, preparation of the manuscript, and decision to publish. 


\section{Availability of data and materials}

All data generated or analyzed during this study are included in this published article and its supplementary information files.

\section{Ethics approval and consent to participate} Not applicable.

\section{Consent for publication}

Not applicable.

\section{Competing interests}

The authors declare that they have no competing interests.

Received: 16 April 2020 Accepted: 31 October 2020

Published online: 16 November 2020

\section{References}

1. Uyeo S, Kobayashi S. Lycoris alkaloids. XXIV.: isolation and characterization of lycoremine. Pharm Bull. 1953;1:139-42.

2. Liu J, Hu WX, He LF, Ye M, Li Y. Effects of lycorine on HL-60 cells via arresting cell cycle and inducing apoptosis. FEBS Lett. 2004;578:245-50.

3. Havelek R, Martina S, Karel K, Lenka B, Lucie C, Marketa D, Jirina V, Martina R, Lubomir O, Zuzana B. The effect of Amaryllidaceae alkaloids haemanthamine and haemanthidine on cell cycle progression and apoptosis in p53-negative human leukemic Jurkat cells. Phytomedicine. 2014;21:479-90

4. Doskočil I, Hoštálková A, Šafratová M, Benešová N, Havlík J, Havelek R, Kuneš J, Královec K, Chlebek J, Cahlíková L. Cytotoxic activities of Amaryllidaceae alkaloids against gastrointestinal cancer cells. Phytochem Lett. 2015;13:394-8.

5. Hsu BS, Kurita S, Yu ZZ, Lin JZ. Synopsis of the genus Lycoris (Amaryllidaceae). SIDA, Contributions to Botany. 1994;16:301-31.

6. Bose S, Flory WS. A study of phylogeny and karyotype evolution in Lycoris. Nucleus. 1963;6:141-56.

7. Kurita S. Variation and evolution in the karyotype of Lycoris, Amaryllidaceae VI. Intrapopulational and/or intraspecific variation in the karyotype of $L$. sanguinea max. Var. kiushiana and L. sanquinea max. Var. koreana (Nakai) Koyama. Cytologia. 1988;53:307-21.

8. Kurita S. Variation and evolution on the karyotype of Lycoris, Amaryllidaceae VII. Modes of karyotype alteration within species and probable trend of karyotype evolution in the genus. Cytologia. 1988;53:323-35.

9. Kurita S, Hsu PS. Hybrid complexes in Lycoris Amaryllidaceae. Am J Bot. 1996;83:207.

10. Zhou SB, Yu BQ, Luo Q, Hu JR, Bi D. Karyotype of six populations of Lycoris radiata and discovery of the tetraploid. Acta Phytotaxon Sin. 2007:45:513-22.

11. Liu YX, Zheng YH, Xia T, Zhou J. Karyotype studies on Lycoris radiata populations from China. Genet Mol Res. 2016;15:15017357.

12. Shao JZ, Yang JG, Zhang DC, Nie LW. The discovery of diploid Lycoris radiata (L'her.) herb. From AnHui. Acta Phytotaxon Sin. 1994;6:549-52.

13. Qin WH, Zhou SB, Wang HY. A new chromosome number and karyotype in Lycoris radiata in Anhui Province. Guihaia. 2004;1:29-32 (in Chinese).

14. Kurita S. Variation and evolution on the karyotype of Lycoris, Amaryllidaceae II. Karyotype analysis of ten taxa among which seven are native to China. Cytologia. 1987;52:19-40.

15. Jiang YM. Xu S, Han XK, Wang, HE J, Xia B, Wang R. investigation of nuclear DNA contents of Lycoris species (Amaryllidaceae) with different chromosome number by flow Cytometry. Pak J Bot. 2017:49:2197-200.

16. Joshi SP, Ranjekar PK, Gupta VS. Molecular markers in plant genome analysis. Curr Sci India. 1999;77(2):230-40.

17. Kumar P, Gupta VK, Misra AK, Modi DR, Pandey BK. Potential of molecular markers in plant biotechnology. Plant Omics. 2009;2:141.

18. Powell W, Machray GC, Provan J. Polymorphism revealed by simple sequence repeats. Trends Plant Sci. 1996;1:215-22.

19. Varshney RK, Graner A, Sorrells ME. Genic microsatellite markers in plants: features and applications. Trends Biotechnol. 2005;23:48-55.

20. Li YC, Korol AB, Fahima T, Nevo E. Microsatellites within genes: structure, function, and evolution. Mol Biol Evol. 2004;21:991-1007.

21. Taheri S, Abdullah TL, Rafii MY, Harikrishna JA, Werbrouck SPO, Teo CH, Sahebi M, Azizi P. De novo assembly of transcriptomes, mining, and development of novel EST-SSR markers in Curcuma alismatifolia (Zingiberaceae family) through Illumina sequencing. Sci Rep. 2019;9:3047.
22. Xu M, Liu X, Wang JW, Teng SY, Shi JQ, Li YY, Huang MR. Transcriptome sequencing and development of novel genic SSR markers for Dendrobium officinale. Mol Breeding. 2017;37:18.

23. Jia HX, Yang HF, Sun P, Jb L, Zhang J, Guo YH, Han XJ, Zhang GS, Lu MZ, $\mathrm{Hu}$ JJ. De novo transcriptome assembly, development of EST-SSR markers and population genetic analyses for the desert biomass willow, Salix psammophila. Sci Rep. 2016;6:39591.

24. Guo Q, Wang JX, Su LZ, Lv W, Sun YH, Li Y. Development and Evaluation of a Novel Set of EST-SSR Markers Based on Transcriptome Sequences of Black Locust (Robinia pseudoacacia L.). Genes. 2017:8:177.

25. He QL, Shen Y, Chen Y, Zhou Y, Berg A, Wu RL, Huang MR. Development of 16 polymorphic simple sequence repeat markers for Lycoris longituba from expressed sequence tags. Mol Ecol Resour. 2009;9:278-80.

26. Wang $R$, Xu S, Jiang YM, Jiang JW, Li XD, Liang LJ, He J, Peng F, Xia B. De novo sequence assembly and characterization of Lycoris aurea transcriptome using GS FLX titanium platform of 454 pyrosequencing. PLoS One. 2013;8:e60449.

27. Wang $R$, Xu S, Wang N, Xia B, Jiang YM, Wang R. Transcriptome analysis of secondary metabolism pathway, transcription factors, and transporters in response to methyl jasmonate in Lycoris aurea. Front Plant Sci. 2017;7:1971.

28. Chang $L$, Chen J, Xiao Y, Xia Y. De novo characterization of Lycoris sprengeri transcriptome using Illumina GA II. Afr J Biotechnol. 2011;10:12147-55.

29. Shi Y, Tong ZK, Gao YH. Development of EST-SSR markers and genetic diversity analysis in Lycoris sprengeri. J Nucl Agric Sci. 2018;32:1089-96 (in Chinese).

30. Xuan SQ, Zheng JY, Wang H, Gao P, Zhou SB, Liu K, Zhu GP. Polymorphic microsatellite loci for the genetic analysis of Lycoris radiata (Amaryllidaceae) and cross-amplification in other congeneric species. Genet Mol Res. 2011;10:3141-5.

31. Chen IJ, Shii CT, Chang TL, Kang HK. Development of 17 novel microsatellite markers for Lycoris aurea and L. radiata (Amaryllidaceae) using nextgeneration sequencing. Appl. Plant Sci. 2018;6:e1198.

32. González M, Salazar E, Castillo J, Morales P, Mura-Jornet I, Maldonado J, Silva H, Carrasco B. Genetic structure based on EST-SSR: a putative tool for fruit color selection in Japanese plum (Prunus salicina L.) breeding programs. Mol Breeding. 2016;36:68.

33. Gao $Y H$, Zhu YQ, Tong ZK, Xu ZY, Jiang XF, Huang CH. Analysis of genetic diversity and relationships among genus Lycoris based on start codon targeted (SCOT) marker. Biochem Syst and Ecol. 2014;57:221-6.

34. Shi SD, Qiu YX, Li EX, Wu L, Fu CX. Phylogenetic relationships and possible hybrid origin of Lycoris species (Amaryllidaceae) revealed by ITS sequences. Biochem Genet. 2006:44:198-208.

35. Wang ZY, Fang BP, Chen JY, Zhang XJ, Luo ZX, Huang LF, Chen XL, Li YJ. De novo assembly and characterization of root transcriptome using Illumina paired-end sequencing and development of cSSR markers in sweetpotato (Ipomoea batatas). BMC Genomics. 2010;11:726.

36. Wei WL, Qi XQ, Wang LH, Zhang YX, Hua W, Li DH, Li HX, Zhang XR. Characterization of the sesame (Sesamum indicumL.) global transcriptome using Illumina paired-end sequencing and development of EST-SSR markers. BMC Genomics. 2011;12:451.

37. Bazzo BR, Carvalho LD, Carazzolle MF, Pereira GAG, Colombo CA. Development of novel EST-SSR markers in the macaúba palm (Acrocomia aculeata) using transcriptome sequencing and cross species transferability in Arecaceae species. BMC Plant Biol. 2018;18:276.

38. Yan ZZ, Wu F, Luo K, Zhao YF, Yan Q, Zhang YF, Wang YR, Zhang JY. Crossspecies transferability of EST-SSR markers developed from the transcriptome of Melilotus and their application to population genetics research. Sci Rep. 2017;7:17959.

39. Barboza K, Beretta V, Kozub PC, Salinas C, Morgenfeld MM, Galmarini CR, Cavagnaro PF. Microsatellite analysis and marker development in garlic: distribution in EST sequence, genetic diversity analysis, and marker transferability across Alliaceae. Mol Gen Genomics. 2018;293:1091-106.

40. Bostein D, White RL, Skolnick M, David RW. Construction of a genetic linkage map in man using restriction fragment length polymorphism. Am J Hum Genet. 1980:32:314-31.

41. Zeng J, Chen J, Kou YX, Wang YJ. Application of EST-SSR markers developed from the transcriptome of Torreya grandis (Taxaceae), a threatened nutyielding conifer tree. Peer J. 2018;6:e5606.

42. Sathyanarayana N, Pittala R, Tripathi PK, Chopra R, Singh HR, Belamkar V, Bhardwaj PK, Doyle JJ, Egan AN. Transcriptomic resources for the medicinal legume Mucuna pruriens: de novo transcriptome assembly, annotation, identification and validation of EST-SSR markers. BMC Genomics. 2017;1 8:409.

43. Ye YJ, Feng L, Liang XH, Liu TT, Cai M, Cheng TR, Wang J, Zhang QX, Pan HT. Characterization, validation, and cross-species transferability of newly 
developed EST-SSR markers and their application for genetic evaluation in crape myrtle (Lagerstroemia spp). Mol Breeding. 2019;39:26.

44. Groot SPC, Bniinsma J, Karssen CM. The role of endogenous gibberellin in seed and fruit development of tomato: studies with a gibberellin-deficient mutant. Physiol Plantrum. 1987;71:184-90.

45. Ru P, Xu L, Ma H, Huang $H$. Plant fertility defects induced by the enhanced expression of microRNA167. Cell Res. 2006;16:457-65.

46. Schwechheimer C. Understanding gibberellic acid signaling-are we there yet? Curr Opin Plant Biol. 2008;11:9-15.

47. Thiel T, Michalek W, Varshney R, Graner A. Exploiting EST databases for the development and characterization of gene-derived SSR-markers in barley (Hordeum vulgare L.). Theor Appl Genet. 2003;106:411-22.

48. Yeh FC. Population genetic analysis of codominant and dominant markers and quantitative traits. Belg J Bot. 1997;129:157.

49. Nagy S, Poczai P, Cernák I, Gorji AM, Hegedus G, Taller J. PICcalc: an online program to calculate polymorphic information content for molecular genetic studies. Biochem Genet. 2012;50:670-2.

50. Falush D, Stephens M, Pritchard JK. Inference of population structure using multi-locus genotype data: linked loci and correlated allele frequencies. Genetics. 2003;164:1567-87.

51. Pritchard JK, Stephens M, Donnelly P. Inference of population structure using multi-locus genotype data. Genetics. 2000;155:945-59.

52. Evanno $G$, Regnaut $S$, Goudet J. Detecting the number of clusters of individuals using the software STRUCTURE: a simulation study. Mol Ecol. 2005;14:261-2620.

\section{Publisher's Note}

Springer Nature remains neutral with regard to jurisdictional claims in published maps and institutional affiliations.

Ready to submit your research? Choose BMC and benefit from:

- fast, convenient online submission

- thorough peer review by experienced researchers in your field

- rapid publication on acceptance

- support for research data, including large and complex data types

- gold Open Access which fosters wider collaboration and increased citations

- maximum visibility for your research: over $100 \mathrm{M}$ website views per year

At $\mathrm{BMC}$, research is always in progress.

Learn more biomedcentral.com/submissions 